\title{
Leveraging the polymer glass transition to access thermally-switchable shear jamming suspensions
}

Chuqiao Chen

University of Chicago

Mike van de Naald

University of Chicago

Abhinendra Singh

University of Chicago

Neil Dolinski

University of Chicago https://orcid.org/0000-0002-2160-8811

Grayson Jackson

University of Chicago https://orcid.org/0000-0003-0663-3274

Heinrich Jaeger

University of Chicago

Stuart Rowan

University of Chicago https://orcid.org/0000-0001-8176-0594

Juan de Pablo ( $\square$ depablo@uchicago.edu )

University of Chicago https://orcid.org/0000-0002-3526-516X

\section{Article}

Keywords:

Posted Date: February 11th, 2022

DOI: https://doi.org/10.21203/rs.3.rs-1193286/v1

License: (c) (i) This work is licensed under a Creative Commons Attribution 4.0 International License.

Read Full License 


\section{Leveraging the polymer glass transition to access thermally-}

\section{2 switchable shear jamming suspensions}

3 Authors: Chuqiao Chen ${ }^{1}$, Michael van de Naald ${ }^{2}$, Abhinendra Singh ${ }^{1,3}$, Neil D. Dolinski ${ }^{1}$,

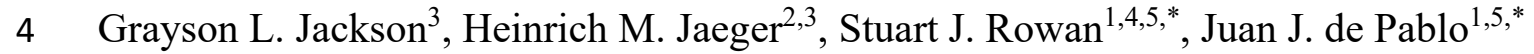

$5 \quad{ }^{1}$ Pritzker School of Molecular Engineering, University of Chicago, Chicago, IL, USA

$6 \quad{ }^{2}$ Department of Physics, The University of Chicago, Chicago, IL, USA

$7 \quad{ }^{3}$ James Franck Institute, The University of Chicago, Chicago, IL, USA

$8 \quad{ }^{4}$ Department of Chemistry, The University of Chicago, Chicago, IL, USA

$9{ }^{5}$ Center for Molecular Engineering, Argonne National Laboratory, Lemont, IL, USA

10 Abstract

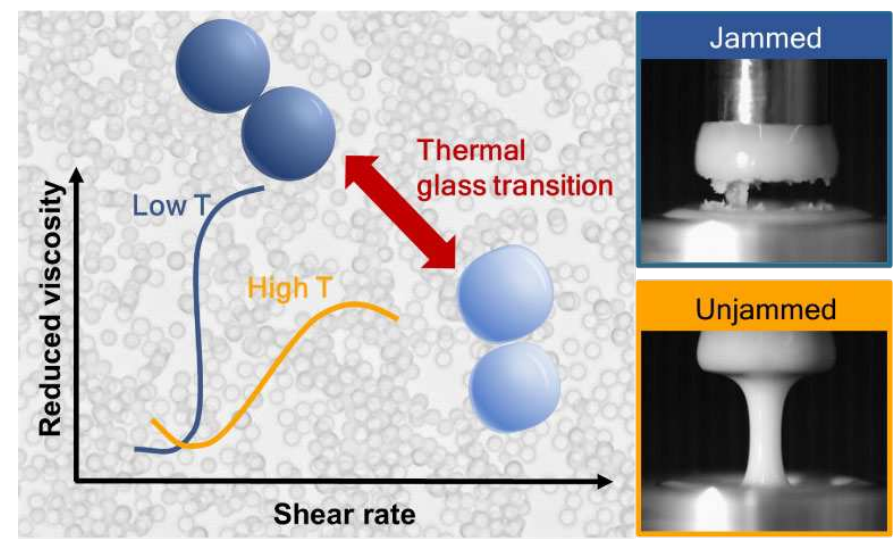

12 When sheared sufficiently strongly, suspensions having a large volume fraction of solid particles can exhibit a dramatic increase in viscosity (shear thickening) and even solidify (shear jamming).

14 Investigations to date have almost exclusively focused on rigid, non-responsive particles, which

15 do not allow in situ tuning of shear thickening or jamming. Here we report suspensions of polymeric micron-sized particles with accessible glass transition temperatures $\left(T_{\mathrm{g}}\right)$ designed to

17 control their non-Newtonian rheology. The underlying mechanical stiffness and interparticle friction between particles change dramatically near $T_{\mathrm{g}}$. We capitalize on these properties and show that, in contrast to conventional systems, a dramatic and non-monotonic change in shear thickening is observed as the suspensions transition through the particles' $T_{\mathrm{g}}$. This behavior enables the in situ turning on or off of shear jamming by varying the temperature relative to $T_{\mathrm{g}}$,

22 and lays the groundwork for thermally switchable jamming systems. 


\section{Introduction}

24 Discontinuous shear thickening (DST) in dense suspensions is a phenomenon in which a sheared

25 fluid experiences a large, abrupt jump in viscosity $(\eta)$, which potentially can lead to solid-like shear-jamming (SJ) behavior ${ }^{1-3}$. This shear-induced transformation from an easily flowing state to a state of much enhanced viscosity is fully reversible, and has been leveraged in a range of applications, including flexible stab-proof armor, smart speed bumps, and damping systems for impact mitigation $^{4-8}$. Although the phenomenon is well-known (e.g., corn starch and water ${ }^{9}$ ), an understanding of the microscopic mechanism behind this transformation has only been developed recently. Under shear, the particles (which typically occupy more than $50 \%$ of the fluid volume, $\phi>50 \%$ ) are forced into sufficiently close proximity with neighbors that they start to interact through direct frictional contacts ${ }^{1,3,10-13}$, causing the emergence of an extended network of frictional force chains that resist further deformation.

To date, most studies of suspensions exhibiting DST or SJ have been restricted to relatively simple chemistries, in which the particles act as rigid, non-deformable objects in relation to the applied shear stress. Typical materials for the particles include silica ${ }^{11,14}$, glassy polymers like poly(methyl methacrylate) (PMMA) $)^{6,15}$ or polystyrene (PS $)^{16,17}$, or rigid non-spherical granular materials like cornstarch ${ }^{9}$. In such systems, key factors that determine DST and SJ include applied stress $(\tau)$, volume fraction $(\phi)$, and interparticle friction ${ }^{3,14,15,17-23}$.

41 For strong shear thickening and shear jamming, where $\phi$ is high and frictional contact forces are crucial, recent studies have shown that the chemical composition and structure of the particle surfaces can affect contact interactions and drastically influence the rheological behavior ${ }^{14,15,19,21}$. While these works and related studies ${ }^{12,24,25}$ have contributed towards a better understanding of the structure-property relationship during DST and SJ, little is known about the roles of particle and interfacial compliance.

47 A considerable amount of research has been aimed at characterizing polymeric interfaces, including molecular mobility and friction, as a material goes through a glass transition. As a 49 polymeric material is heated above its glass transition temperature $\left(T_{\mathrm{g}}\right)$, within a span of just a 50 few degrees, molecular mobility increases by over ten orders of magnitude ${ }^{26-28}$, elastic moduli 51 drop dramatically ${ }^{28,29}$, and friction exhibits a distinct temperature anomaly ${ }^{29-35}$. Building on that 
52 body of work, this work considers the interplay between polymer glass formation/devitrification 53 and shear-jamming phenomena, and how that can be leveraged to arrive at suspensions with 54 adaptable shear-jamming characteristics. More specifically, we introduce a generally applicable strategy that relies on design of suspensions of particles with targeted $T_{\mathrm{g}}$, and we investigate the impact of the $T_{\mathrm{g}}$ on their shear rheology. At temperatures below $T_{\mathrm{g}}$ the particles are rigid, with a tensile storage modulus $\left(E^{\prime}\right)$ on the order of $10^{9} \mathrm{~Pa}$ (close to that of hard spheres). Above $T_{\mathrm{g}}$, the particles become rubbery and deformable, with $\mathrm{E}^{\prime}$ decreasing to $\sim 10^{6} \mathrm{~Pa}$. It is hypothesized that such a pronounced change in stiffness around $T_{\mathrm{g}}$ will impact interparticle contact interactions and force-chain rigidity, thereby providing a versatile platform for tunable shear thickening and shear 61 jamming.

\section{Results and Discussions}

While there are many examples of polymeric particles reported in the dense suspension literature, most are based on poly(styrene) (PS) or poly(methyl methacrylate) (PMMA), which exhibit a glass transition temperature $\left(T_{\mathrm{g}}\right)$ that is well above ambient conditions $\left(T_{\mathrm{g}} \sim 100^{\circ} \mathrm{C}\right)$ and rarely accessed in rheological measurements ${ }^{6,15,16,36}$. Furthermore, non-crosslinked PS or PMMA particles can suffer from irreversible plastic deformations and dissolution above $T_{\mathrm{g}}{ }^{37,38}$, and randomly crosslinked polymers can exhibit broadened $T_{\mathrm{g}}{ }^{\prime} \mathrm{s}$ that can be difficult to characterize ${ }^{39-}$ 41. These and other features serve to underscore that studying the effect of $T_{\mathrm{g}}$ in suspension rheology requires careful choice of the particles' chemistry.

To access a series of crosslinked particles with different and accessible $T_{\mathrm{g}} \mathrm{s}$, polymeric particles were synthesized using thiol-Michael dispersion polymerization ${ }^{42-44}$ of small molecule monomers (Fig. 1a,b). This technique offers several advantages: (1) the product is a polymer network with high crosslinking density and minimal plastic deformation above $T_{\mathrm{g}}$, (2) the glass transition temperature range is narrow, (3) the $T_{\mathrm{g}}$ of the polymer can be precisely tuned by

77 varying the monomer structures, and (4) the reaction is facile and relatively insensitive to moisture or air ${ }^{42-44}$. Three types of particles (referred to as $\mathbf{P}-\mathbf{X}$, where $\mathrm{X}$ is the dry $T_{\mathrm{g}}$ in ${ }^{\circ} \mathrm{C}$ ), $\mathbf{P}$ - 
80 thiol/vinyl ratio following general procedures reported by Bowman and coworkers ${ }^{42,43}$. In each

81 case, a tetrathiol, pentaerythritol tetrakis(3-mercaptopropionate) (PETMP, 1), was mixed with

82 monomer(s) containing multiple Michael acceptor functionalities, trimethylolpropane triacrylate

83 (TMPTA, 2), divinyl sulfone (DVS, 3), or 1,3,5-triacryloylhexahydro-1,3,5-triazine (TAHTZ, 4),

84 at an alkene to thiol ratio of 1:1. By varying the type and ratio of the different Michael acceptor-

85 containing monomers it was possible to tailor the $T_{\mathrm{g}}$ of the particles (Fig. 1c-e). The reaction was

86 monitored by alkene/thiol conversion via FTIR spectroscopy (see Supplementary Fig. S1-S3).

87 Images from scanning electron microscopy (SEM) demonstrate that the particles were uniform in

88 size (Fig 1.c-e, and Supplementary Fig. S4).

89 To create suspensions, poly(ethylene glycol) of molecular weight $200 \mathrm{~g} / \mathrm{mol}$ (PEG200) was used

90 as the carrier fluid for all the particle systems (in the case of the P-29 particles, 20 vol\%

91 dimethyl sulfoxide was added as a cosolvent, see the Methods section for further details). The $T_{\mathrm{g}}$

92 of the dry particles and the suspensions were determined by differential scanning calorimetry

93 (DSC) as shown in Fig. 1f. In all three cases, the $T_{\mathrm{g}}$ of the particles immersed in the carrier fluid

94 drops by $15 \sim 25^{\circ} \mathrm{C}$ from that of the dry particles, suggesting that the carrier fluid acts as a

95 plasticizer. Nevertheless, the particle size stays roughly the same in the dry or wet state on

96 account of their high crosslinking density (see Supplementary Fig. S5).

97 
a

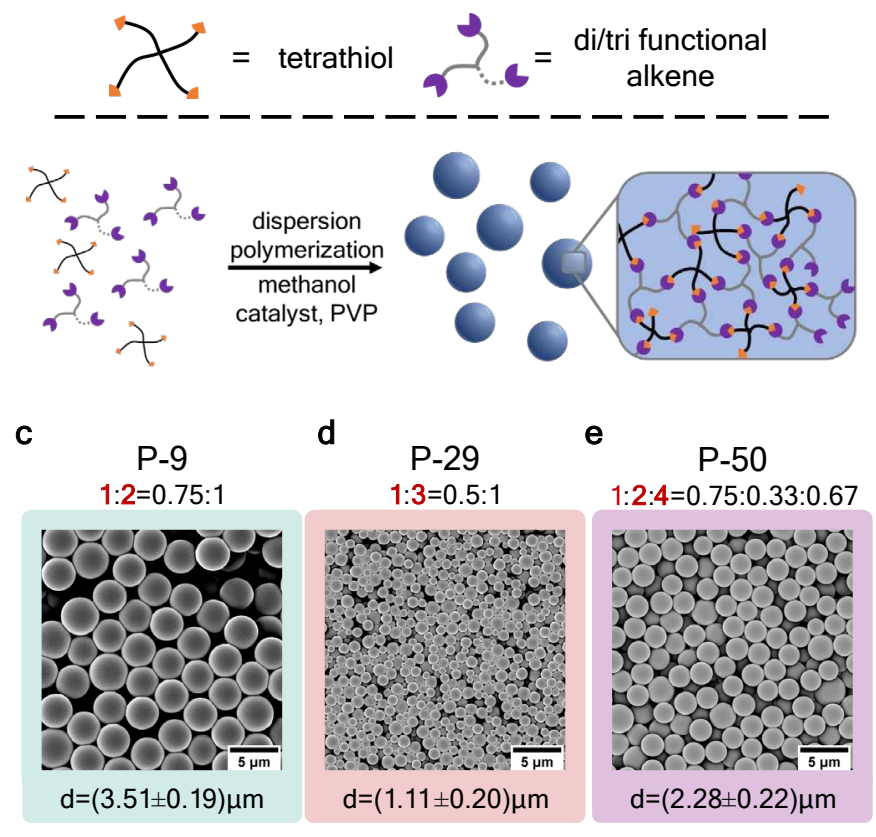

b

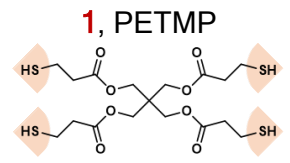

3, DVS
2, TMPTA

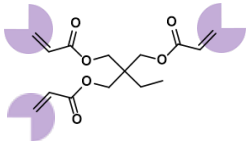

4, TAHTZ

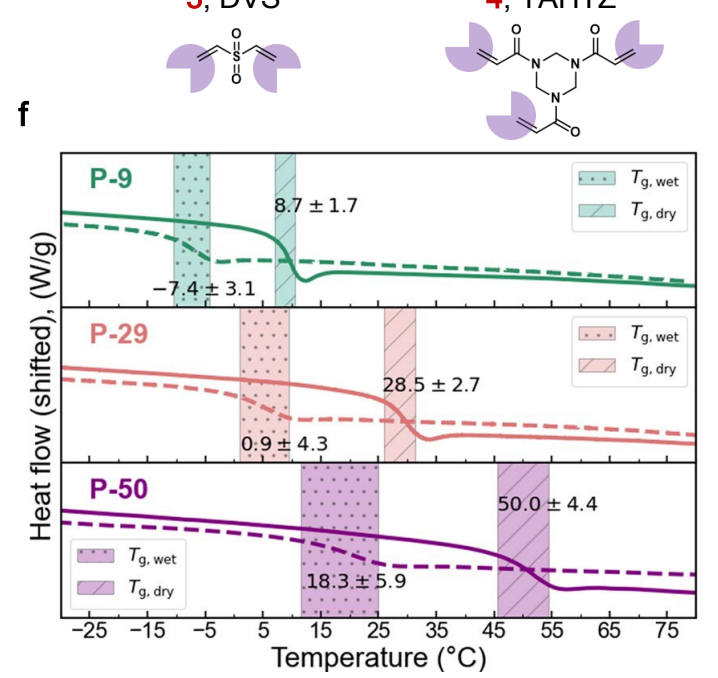

99

100

101

102

103

104

105

106

107

108

109

110

111

112

113

114

115

116

117

118

119

120

Fig. 1 | Particle synthesis scheme and characterization by SEM and DSC. a, Schematic diagram of the Thiol-Michael dispersion reaction and the particle network structure. Detailed reactions and purification conditions are in the Methods section. b. Chemical structures of the monomers, pentaerythritol tetrakis(3-mercaptopropionate) (PETMP, 1), trimethylolpropane triacrylate (TMPTA, 2), divinyl sulfone (DVS, 3), or 1,3,5-triacryloylhexahydro-1,3,5-triazine (TAHTZ, 4), used to synthesize the different particles. c-e, The monomer composition and SEM micrographs of P-9, P-29 and P-50 particles, respectively. The mean size and the standard deviation are indicated below each image. f, DSC measurement results showing the normalized heat flow as a function of $T$ for the dry particles (solid line) and particles suspended in their respective carrier fluid at around $56 \mathrm{wt} \%$ (dashed lines). Shaded areas with (.) and (/) indicate the wet and dry $T_{\mathrm{g}}$ respectively. The first number is the half-height midpoint of the glass transition $\left(T_{\mathrm{g}}\right)$ range. The second value is the width of the $T_{\mathrm{g}}$ range.

As the thermomechanical properties of these networks represent a critical aspect of this work, we evaluated them by preparing P-9, P-29 and P-50 films, which were characterized by dynamic mechanical analysis (DMA) in both the dry (see Supplementary Fig. S6-S7) and carrier-fluidswollen state (Fig. 2) for a better representation of the suspension environment. Consistent with the literature ${ }^{42,43}$, the polymer networks synthesized using this chemistry exhibit a relatively narrow glass transition window. The thermomechanical $T_{\mathrm{g}}$ of the materials, designated by the peak in $\tan \delta$, are comparable to the DSC measurements (Fig. 1f) for the dry and immersed film (see Supplementary Fig. S6-S7). Importantly for these studies, the tensile storage modulus $\left(E^{\prime}\right)$ changes by three orders of magnitude, from around $10^{9} \mathrm{~Pa}$ in the glassy state to $10^{6} \mathrm{~Pa}$ in the rubbery state for all three materials. 


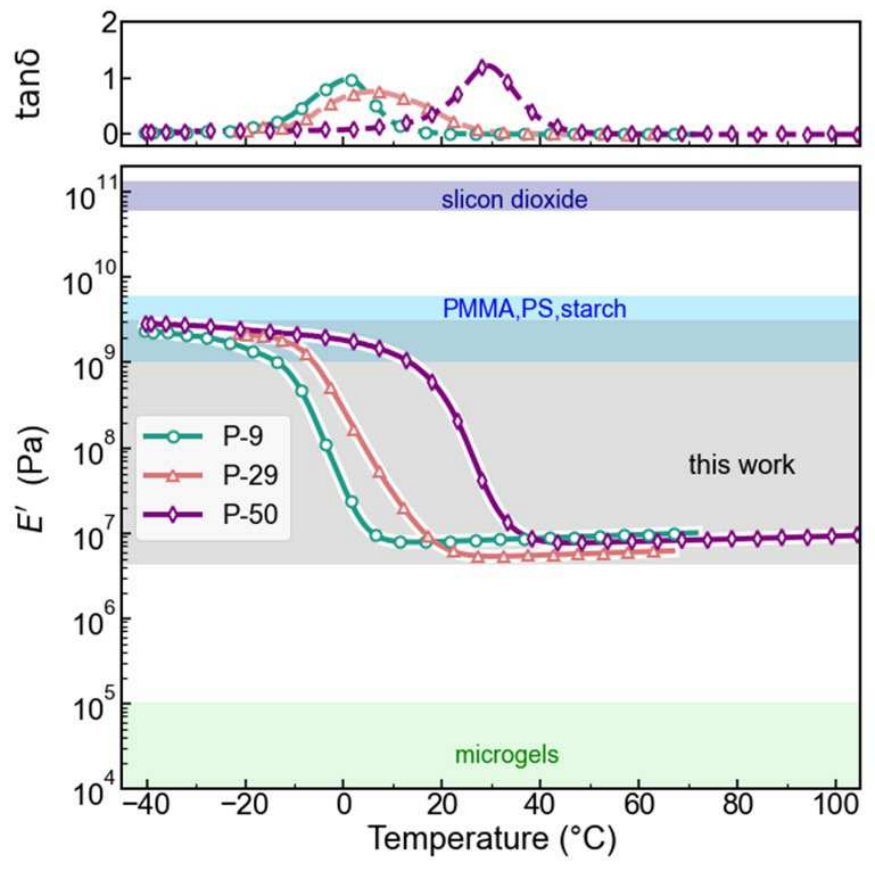

Fig. 2| Mechanical stiffness characterization via DMA. The storage modulus $\left(E^{\prime}\right)$ and $\tan \delta=E^{\prime \prime} / E^{\prime}$ of the carrier fluid-swollen P-9, P-29 and P-50 polymer films were measured in an immersion setup, using an oscillation frequency of $\mathrm{f}=1 \mathrm{~Hz}$. See Supplementary Fig. S6 for plots including loss modulus $\left(E^{\prime \prime}\right)$. Thermomechanical $T_{\mathrm{g}}$ is designated by the peak in $\tan \delta$. The values of $\mathrm{E}^{\prime}$ for several other particle materials are indicated for comparison ${ }^{45-48}$, PMMA: Poly (methyl methacrylate) and PS: polystyrene.

To examine the effect of temperature $(T)$ on rheology, steady-state flow curves for particle suspensions with fixed particle volume fraction were measured at different temperatures as a function of stress $(\tau)$. The suspension viscosity $\eta$ can be normalized by the viscosity $\eta_{0}(T)$ of the Newtonian carrier fluid to define a relative viscosity $\eta_{\mathrm{r}}=\eta / \eta_{0}(T)$ (see Supplementary Fig. S8). Critically, the strength of shear thickening can be parameterized by the power law exponent $\beta\left(\eta_{\mathrm{r}} \propto \tau^{\beta}\right)$, which represents the slope of the curve during the shear thickening regime on a log$\log$ plot, with $\beta=0$ corresponding to a Newtonian fluid; and $\beta=1.0$ signaling the occurrence of discontinuous shear thickening (DST), which is a precursor of shear jamming in steady-state rheological measurements ${ }^{1,9,24}$.

In typical suspensions of hard spheres, such as silica particles, the $\eta_{\mathrm{r}}$ vs $\tau$ curves are independent of temperature, and for a given packing fraction $\beta$ is roughly constant (with an estimated decline of less than 0.03 per $\left.10^{\circ} \mathrm{C}\right){ }^{11,49}$. In contrast to the behavior of these traditional suspensions, we find that the $\mathbf{P - 9}$ particle suspension shows a strong sensitivity to temperature, as demonstrated 
141 in Fig. 3a, where $\eta_{\mathrm{r}}$ for a volume fraction of $\phi=53 \%$ shows a significant change in shear

142 thickening strength as $T$ is varied from $-15^{\circ} \mathrm{C}$ to $45^{\circ} \mathrm{C}$. Here, a strong, nearly discontinuous

143 thickening with $\beta \approx 0.9$ was observed below $15^{\circ} \mathrm{C}$. This should be contrasted with

144 measurements at higher temperatures, for example $35^{\circ} \mathrm{C}$ and $45^{\circ} \mathrm{C}$, where the suspension shows

145 comparatively mild thickening $(\beta<0.7)$ (Fig. 3b and Supplementary Fig. S9a). This change in

$146 \beta$ over a temperature range of $\sim 20^{\circ} \mathrm{C}$ suggests that control of shear thickening should be possible

147 simply by modulating temperature, thereby providing new avenues for engineering responsive

148 fluids or for facilitating suspension processing.
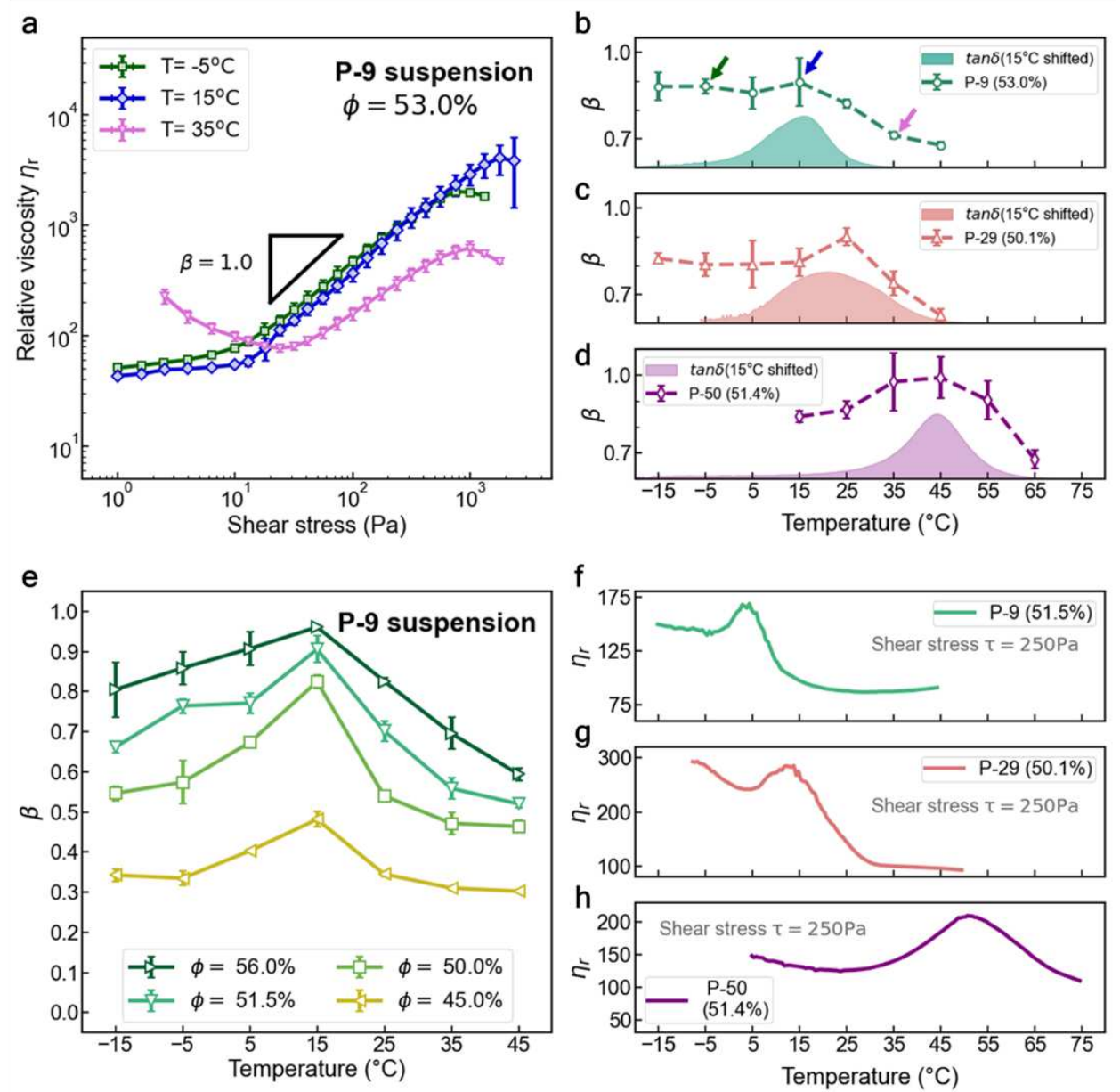

Fig. 3 | Dependence of rheological behavior on temperature. a, Stress-controlled steady-state rheometry data for the P-9 suspension with $\phi=53.0$ vol\% at $-5^{\circ} \mathrm{C}$ (green), $15^{\circ} \mathrm{C}$ (blue) and $35^{\circ} \mathrm{C}$ (pink), demonstrating that the strength of shear thickening is highly temperature dependent. The black line has a slope of 1 corresponding to DST where the shear rate is constant. A plot of $\eta_{\mathrm{r}}$ vs shear rate can be found in the Supplementary Fig. S9. b-d, $\beta$ as a function of 53.0\% P-9 suspension, 50.1\% P-29 suspension, $51.4 \%$ P-50 suspension. See Supplementary Fig. S9 for the flow curves. Shaded areas indicate the tan $\delta$ results from Fig. 2 , here shifted up by $15^{\circ} \mathrm{C}$. Arrows in $\mathbf{b}$ indicate $\beta$ for the conditions measured in a. e, Plots of the shear thickening exponent $\beta\left(\eta_{r} \propto \tau^{\beta}\right)$ as a function of temperature for all seven packing 
fractions of P-9 suspensions. See Supplementary Fig. S10 for the flow curve. Error bars represent the standard deviation from three or more replicate measurements in a-e. f-h, Reduced viscosity $\eta_{\mathrm{r}}$ measured as a function of temperature for 51.5\% P-9 suspension, 50.1\% P-29 suspension, 51.4\% $\mathbf{P}-\mathbf{5 0}$ suspension at a shear stress of $250 \mathrm{~Pa}$. Plots of $\eta$ vs $T$ for the cooling and heating steps can be found in Supplementary Fig. S12.

To test whether this phenomenon is indeed related to the particles' $T_{\mathrm{g}}$, further experiments were carried out on suspensions of P-29 and P-50. While the three types of particles have different sizes (ranging from ca. 1 to $3.5 \mu \mathrm{m}$ ), the effect of particle size is well understood, and has been shown to mainly affect the onset shear stress of thickening, but not the trend in $\beta^{3,50}$. For these higher $T_{\mathrm{g}}$ suspensions, the non-monotonic behavior in $\beta$ is found to be similar for all particle compositions. The peak, however, is shifted to higher temperatures, consistent with the changes in $T_{\mathrm{g}}$ (Fig. 3b-d, see Supplementary Fig. S9 for the flow curves). Critically, in all three cases the peak in $\beta$ can be well aligned with $\tan \delta$ in Fig. 2 when the latter is shifted by the same amount $\Delta T=15^{\circ} \mathrm{C}$, suggesting that the temperature dependence is directly related to the thermal glass transition of the particles. An offset $\Delta T$ is consistent with literature data, where polymer interfaces under confinement and, in particular, for different deformation rates, can exhibit dynamics different from the bulk ${ }^{28,51}$. This result motivates further research to quantify how polymer stress relaxation at the interface can influence the constraints on particle relative motions $^{3}$. For the three materials considered here, the fact that the maximum observed $\beta$ strongly tracks with $T_{\mathrm{g}}$ offers a general, yet simple approach for controlling the shear thickening characteristics of dense suspensions by simply tuning the $T_{\mathrm{g}}$ of the particles.

To explore how $\beta$ vs. $T$ varies for different volume fraction $(\phi)$, detailed studies were carried out using P-9 suspensions at four additional $\phi$ values that span weak to strong shear thickening regimes (see Fig. 3 e and Supplementary Fig. S10). In all cases, $\beta$ is a non-monotonic function of $T$, with a peak near $15^{\circ} \mathrm{C}$ for all volume fractions studied (Fig. 3e). Above $15^{\circ} \mathrm{C}, \beta$ drops significantly as the temperature increases. At $45^{\circ} \mathrm{C}, \beta$ is smaller than 0.7 even at highest $\phi$ studied, implying that DST has been suppressed. At this temperature, the elastic modulus is around 3 orders of magnitude smaller than in the glassy state. In this rubbery regime, the deformation caused by typical interparticle stress in shear experiments is estimated to be around $1 \sim 3 \%$ of the particle radius (see Supplementary Fig.S11 for experimental evidence of deformability and additional calculation details). Although this number appears small in 
magnitude, it is about 100 times larger compared to the deformation in the glassy state and may affect the constraints on particle motions. We therefore speculate that the change in deformability may lead to the observed attenuation in shear thickening at temperatures much above $T_{\mathrm{g}}$ : as temperature increases, the particle surfaces become more compliant, discouraging the formation of sample spanning rigid force chains under shear ${ }^{13,52}$.

As an alternative to measuring $\beta$ vs $T$, a common way to probe molecular dynamics in polymer and molecular glass transition is to investigate how viscosity varies with temperature for a given stress $^{53}$. A temperature ramp experiment under constant shear stress ( $\tau=250 \mathrm{~Pa}$, heating/cooling rate $=1.5^{\circ} \mathrm{C} / \mathrm{min}$ ) was conducted for P-9, P-29 and $\mathbf{P}-50$ suspensions (Fig. $3 \mathrm{f}-\mathrm{h}$ and Supplementary Fig. S12). In all cases, particle volume fraction was held at $\phi \sim 50 \%$, since intermediate $\phi$ shows the most prominent peak in $\beta$ in Fig. 3e. To normalize the temperature dependency of carrier fluid viscosity $\left(\eta_{0}\right), \eta_{\mathrm{r}}=\eta / \eta_{0}(T)$ is shown in Fig. $3 \mathrm{f}-\mathrm{h}$. The relative viscosity $\eta_{\mathrm{r}}$ can be regarded as a measure of the additional resistance due to the presence of the particles. For conventional hard sphere suspensions, the curve is expected to be approximately flat with a slight decreasing trend with temperature ${ }^{11,49}$. For all systems measured in this study, it was found that the relative viscosity of the suspension clearly shows a local peak near each particle's $T_{\mathrm{g}}$, which further supports that the maximal shear thickening occurs around the glass transition. Interestingly, the observed behavior in viscosity bears similarities to studies of polymer dispersions that undergo microphase separation, such as in block copolymer solutions ${ }^{54}$ and polymers around their lower critical solubility temperature (LCST) ${ }^{55}$. In those studies, a peak in $\eta$ is observed at a temperature where the conformation of polymer chain is changing. Here, the peak in $\eta_{\mathrm{r}}$ suggests maximal constraints on the suspension flow. It is worth noting that this peak can be observed in both heating and cooling experiments (Supplementary Fig. S12), implying reversibility in the interactions induced by $T_{\mathrm{g}}$.

The observed non-monotonicity leads us to consider other effects of temperature on the suspension. First, the thermal expansion coefficients $(\alpha)$ of the particle materials was evaluated since if $\phi$ increases significantly near $T_{\mathrm{g}}$ then $\beta$ should also increase ${ }^{11,24}$. However, it was found that $\alpha$ of the particles is comparable to that of the carrier fluid (see Supplementary Fig. S13). For all suspensions, the calculated drift in $\phi$ is minimal $\left(\Delta \phi<0.05 \%\right.$ for changing $T$ by $\left.10^{\circ} \mathrm{C}\right)$ and therefore unlikely to be the main cause of the peak in $\beta$ (see Supplementary Fig. S14). 
220 Surface friction, which is of vital importance for constraining particle motion, is also extremely

221 sensitive to temperature in the vicinity of $T_{\mathrm{g}}{ }^{29-35}$. Different from hard surfaces, friction between

222 polymer surfaces originates from dissipative interactions of interdigitating chains ${ }^{30,31,33,35}$.

223 Around $T_{\mathrm{g}}$, adhesive interactions increase on account of an increase in true contact area, which

224 may lead to stronger cohesive 'sticky' forces and enhanced rolling friction ${ }^{21,31,32}$. On account of

225 the viscoelastic nature of the interface between two particles sheared into contact, the friction

226 coefficient $(\mu)$ can either increase monotonically with $T$ at moderate to high surface deformation

227 rates, or show a peak if the deformation rate is slow enough to cross over into the time scale of

228 surface relaxation ${ }^{32-35}$. In relation to shear thickening, simulations have shown that both higher

229 friction coefficients $\mu$ or the introduction of rolling friction could lead to more pronounced shear

230 thickening and larger $\beta^{12}$.

231 We suggest that the non-monotonic trend in the strength of shear thickening with temperature

232 (Fig. 3b-h) results either from a non-monotonic $\mu$ or from a competition between increasing $\mu$

233 and decreasing mechanical stiffness. In both scenarios, shear thickening is most pronounced

234 when relative particle movement is most constrained. A proposed temperature dependency

235 mechanism is shown in Fig. 4.

236 It is worthwhile to consider the findings reported here in the context of devitrification, to results

237 from a different strategy by Hsu et al. ${ }^{21}$, in which polymers that can undergo a lower critical

238 solution temperature (LCST) transition ${ }^{21}$ were grafted onto hard silica particles. These authors

239 also report that adhesion and friction forces can be tuned as a function of temperature, thereby

240 influencing shear thickening. In our particles, the variation of apparent stiffness with temperature

241 is opposite to the effects of an LCST transition, and the non-monotonic trend in $\beta$ suggests

242 previously unconsidered effects of surface deformability in addition to the effects related to $\mu$.

243 Unlike the LCST transition, where the carrier fluid is limited to water and ionic liquids ${ }^{56}$, a

244 strategy relying on $T_{\mathrm{g}}$ is potentially applicable to any carrier fluid. 

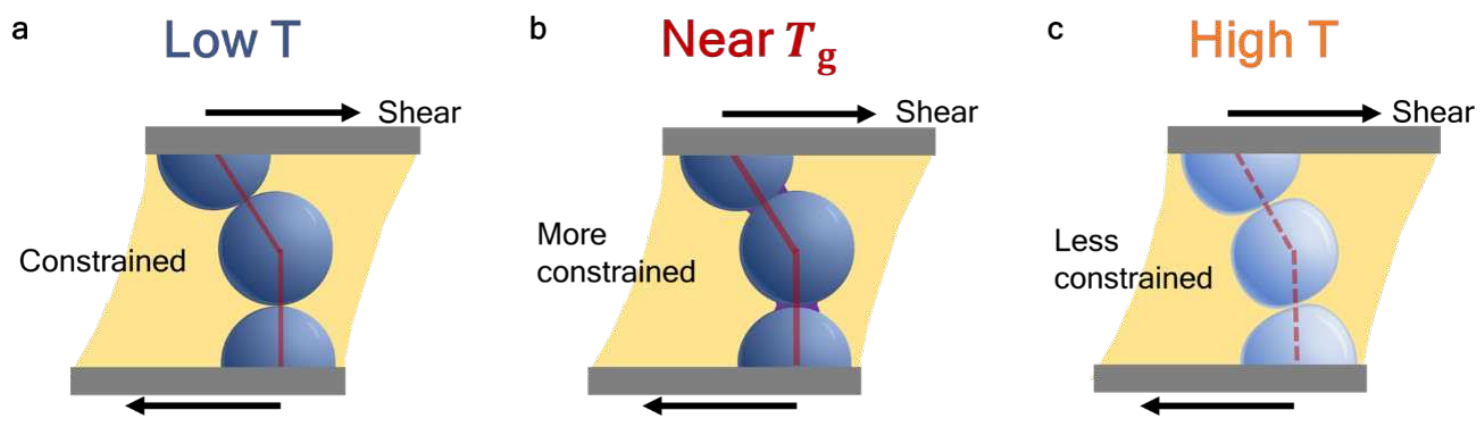

Fig. 4| Illustration of the proposed temperature dependency mechanism. a, Below $T_{\mathrm{g}}$, the particles are glassy and non-deformably. $\mathbf{b}$, The particles exhibit maximum constraints near $T_{\mathrm{g}}$ due to greater frictional interactions between the polymer particles. c, At temperatures high above $T_{\mathrm{g}}$ surface deformability dominates and the particles are less constrained. The deformations are small, as estimated from contact mechanics calculations (Supplementary materials and Fig. S11), and they are exaggerated in this illustration for visual clarity.

Finally, it is important to recall that suspensions that undergo strong or even discontinuous shear thickening in a steady-state rheological measurement may not exhibit shear jamming 9,15 . To directly assess if our design strategy based on $T_{g}$ can be used for switching shear jamming on or off at different temperatures, pull tests ${ }^{15,57}$ were carried out using the P-9 and P-50 suspensions. In these experiments, a cylindrical rod, initially immersed in the suspension, is pulled out vertically at a fixed rate, in our case $8 \mathrm{~mm} / \mathrm{s}$. At $5^{\circ} \mathrm{C}$, the $\mathbf{P - 9}$ suspension shows a rough cleavage plane, indicating brittle fracture associated with the solid-like behavior of shear-jammed fluids. In contrast, at $35^{\circ} \mathrm{C}$ and $65^{\circ} \mathrm{C}$, the $\mathbf{P - 9}$ suspension exhibits a neck and pinch-off detachment which is characteristic of a liquid-like response (Fig. 5a). The transition between these two types of behavior is also reflected in the maximum normal force $F_{\max }$ during the deformation, which shows a sharp decrease near $10^{\circ} \mathrm{C}$ for the P-9 system (Fig. 5b, see Supplementary Fig. S15 for the raw force curves). In contrast, $\mathbf{P - 5 0}$ system shows brittle fracture and large $F_{\max }$ all the way up to $45^{\circ} \mathrm{C}$, with liquid-like response occurring only above $45^{\circ} \mathrm{C}$ (Fig. $5 \mathrm{c}$ and Fig. 5d). This clear dependency on $T_{\mathrm{g}}$ demonstrates how the shear jamming response can be tailored by changing the $T_{\mathrm{g}}$ of the particles. 
a

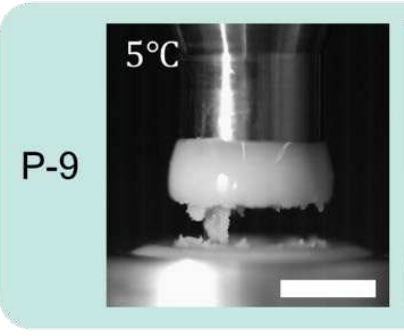

C
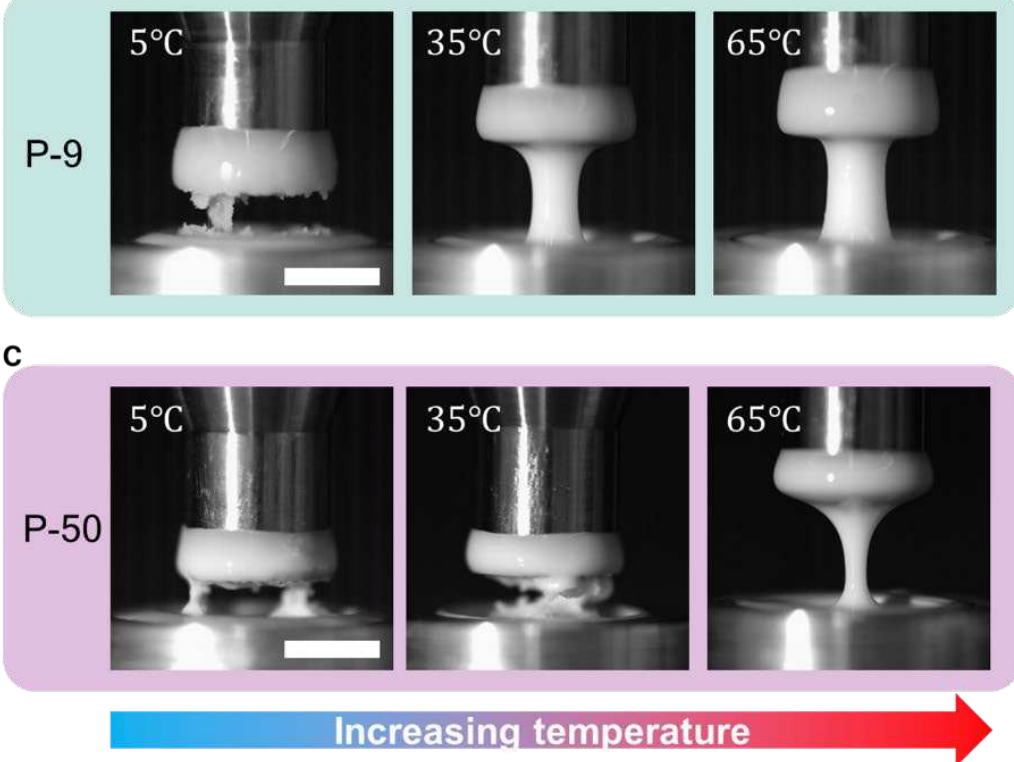

b

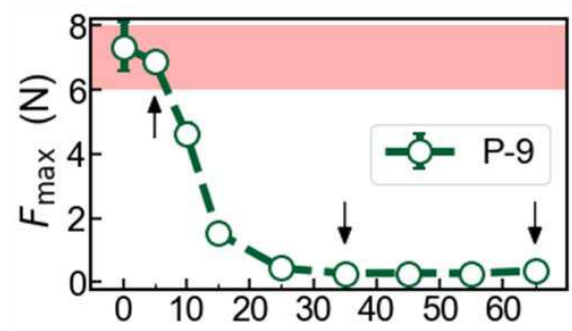

d

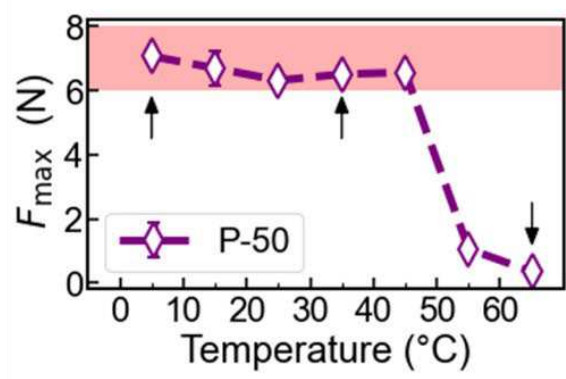

Fig. 5 Tensile testing for shear jamming at varying temperatures. a and $\mathbf{c}$, Images of the suspensions under extensional deformation taken at $T=5^{\circ} \mathrm{C}, 35^{\circ} \mathrm{C}$ and $65^{\circ} \mathrm{C}$ for $\mathbf{P - 9}$ and $\mathbf{P - 5 0}$ suspensions $(\phi=$ $56.0 \%$ ). The pulling rate is $8 \mathrm{~mm} / \mathrm{s}$. The scale bar indicates $5 \mathrm{~mm}$. $\mathbf{b}$ and $\mathbf{d}$, The maximum normal force $\left(\mathrm{F}_{\max }\right)$ as a function of temperature. Shaded areas in red indicate large peak normal forces associated with shear jamming. The arrows indicate temperatures corresponding to the snapshots in a and c. Error bars represent the standard deviation of three replicate measurements. See Supplementary Fig. S15 for representative raw force traces.

\section{Concluding remarks}

In this study, we have demonstrated that a pronounced temperature dependence of the strength of shear thickening in suspensions of polymer microparticles can be achieved by leveraging their glass transition temperature. Most strikingly, the suspensions exhibit maximum shear thickening near $T_{\mathrm{g}}$, which is attributed to enhanced frictional interactions between the polymer particles sheared into contact. At temperatures above $T_{\mathrm{g}}$, the particle surfaces become more deformable and do not constrain relative particle movement as strongly. This directly impacts force chain formation and, macroscopically, the resistance to flow as measured by the suspension viscosity (Fig. 4).

In recent years, there has been a growing interest in designing shear thickening fluids (STFs) for targeted material applications. Here we have shown how the thermal glass transition in polymers can be used in these efforts. Since $T_{\mathrm{g}}$ affords wide tunability by altering the chemical structure of 
the polymer, we believe that this work provides a versatile platform for engineering STFs with tailored mechanical performance.

From a theoretical perspective, this work raises many exciting questions that warrant deeper investigation. For example, the contact mechanics at a polymer-polymer interface are fundamentally different from those at conventional rigid particle surfaces. It remains to be seen how the interfacial polymer dynamics (i.e., various relaxation times at various length scales) affect the formation and destruction of inter-particle force chains. Conversely, given that DST and SJ are exquisitely sensitive to the strength of particle-particle contact interactions, dense suspension rheology provides a powerful lens with which to observe macroscale consequences that are a direct result of interfacial polymer dynamics at molecular length and time scales.

\section{Materials and Methods}

Materials. Pentaerythritol tetrakis(3-mercaptopropionate) (PETMP) ( $>95.0 \%$, Sigma-Aldrich), trimethylolpropane triacrylate (TMPTA) ( $>90 \%$, Sigma-Aldrich), 1,3,5-triacryloylhexahydro1,3,5-triazine (TAHTZ, Sigma-Aldrich), divinyl sulfone ( $>96.0 \%$, TCI America), hexylamine (HEA) ( $>99.0 \%$, Sigma-Aldrich) and triethylamine (TEA) (>99.5\%, Sigma-Aldrich), polyvinylpyrrolidone with average molecular weight 40,000 (PVP) (Sigma Aldrich) were used as received for reactions. Polyethylene glycol with average molecular weight 200 (PEG200) (Sigma Aldrich) and dimethyl sulfoxide (DMSO) (>99.9\%, Sigma-Aldrich) were used as received for dispersing the particles. Solvents including methanol, acetonitrile were purchased from SigmaAldrich and used as received. PPP-LFMR silicon probes were purchased from Asylum Oxford Instruments.

Synthesis of P-9 particles. P-9 particles were synthesized via dispersion polymerization following literature procedures. Monomers $5.93 \mathrm{~g}(20 \mathrm{mmol})$ of TMPTA and $7.33 \mathrm{~g}(15 \mathrm{mmol})$ of PETMP were dissolved in $270 \mathrm{~mL}$ methanol. 3.0g of PVP was dissolved in $30 \mathrm{~mL}$ methanol and transferred to the reaction ask. The mixture of monomers and surfactant was stirred at 400rpm using a mechanical stir. The reaction was started by adding in the base catalyst hexylamine $(0.21 \mathrm{~g}, 1.6 \mathrm{wt} \%$ relative to monomers) which was diluted by 10 times beforehand in methanol. The reaction was carried out for 4 hours at ambient conditions. The base was neutralized by $1 \mathrm{M} \mathrm{HCl}$ aqueous 
solution prior to centrifugation. The dispersion was centrifuged at $1750 \mathrm{~m} / \mathrm{s}^{2}$ for 6 minutes and the supernatant was thrown away. To remove the surfactants and catalyst, the particles were washed in methanol at a concentration of around $0.04 \mathrm{~g} / \mathrm{mL}$ under sonication and vortexing, followed by centrifugation. This washing process was repeated for 3 times. The particles are dried at 0.2 torr for 48 hours and kept in a desiccator before use. The second batch which was used for OM measurement had a smaller particle size distribution averaged around $3.1 \mu \mathrm{m}$. For synthesizing the P-9 particles of $2.5 \mu \mathrm{m}$ used for the temperature ramp experiments, triethylamine (4.0 $\mathrm{wt} \%$ relative to monomers) was used instead.

Synthesis of P-29 particles. P-29 particles were synthesized via dispersion polymerization with the same procedure as the $\mathbf{P}-\mathbf{9}$ particles with catalyst hexylamine $(0.17 \mathrm{~g}, 1.6 \mathrm{wt} \%$ relative to monomers). Monomers were changed to $3.54 \mathrm{~g}$ ( $30 \mathrm{mmol})$ of DVS and $7.33 \mathrm{~g}(15 \mathrm{mmol})$ of PETMP.

Synthesis of P-50 particles. P-50 particles were synthesized via dispersion polymerization with procedure similar to the P-9 particles. The molar ratio of TMPTA to TAHTZ was kept 1:2. Specifically, $1.98 \mathrm{~g}(6.67 \mathrm{mmol})$ of TMPTA, $3.32 \mathrm{~g}(13.33 \mathrm{mmol})$ of TAHTZ and $7.33 \mathrm{~g}(15 \mathrm{mmol})$ PETMP was used. Due to the low solubility of TAHTZ monomer at room temperature, the solvent was preheated to $40^{\circ} \mathrm{C}$ and the monomer was sonicated with the solvent for 1 minute. $0.50 \mathrm{~g}$ of triethylamine was used as catalyst ( $4.0 \mathrm{wt} \%$ relative to the monomers). The rest of the procedures were identical to the synthesis of the $\mathbf{P}-\mathbf{9}$ particles.

Scanning electron microscopy. The particles were solvent casted with methanol on silicon wafers and dried in air. Before the SEM analysis, the particles were sputter coated with a $8 \mathrm{~nm}$ film of $\mathrm{Pt} / \mathrm{Pd}$ metal to enhance electron conductivity. The images were recorded on a Merlin SEM (Carl Zeiss) at a voltage $1.5 \mathrm{kV}$ using Inlens detectors and each image was averaged between 8-15 images. Particle sizes were analyzed with ImageJ.

Optical microscopy. OM images were taken with an optical microscope (Leica DM2700P) using a 50x objective under transmission mode. Particle suspensions were confined in a channel of around $50 \mu \mathrm{m}$ to avoid compression from the cover slip.

Suspension preparation. Dried particles were dispersed in carrier fluid at certain weight percentages. The weight concentration is converted to volume concentration by the densities of the polymer and the fluid. The mixture was stirred extensively and sonicated for 1 hour. For P-9 
and P-50 particles, PEG200 was used as the carrier fluid. For P-29 particles, a mixture containing 80vol\% PEG200, 20vol\% DMSO and $0.5 \mathrm{wt} \% \mathrm{NaCl}$ was used to disperse the particles and screen electrostatic interactions.

Polymer film synthesis. Monomers were dissolved in acetonitrile at $30 \mathrm{wt} \%$ concentration. Triethylamine was added as a catalyst at a concentration of $0.6 \mathrm{wt} \%$ relative to the monomers. After mixing, the solution was immediately injected between two glass slides separated by $1 \mathrm{~mm}$ spacer. The reaction was carried out overnight in solvent atmosphere. To remove the catalyst, the film was washed with methanol and acetone alternately for 4 times. To evaporate the solvent, the film was put in a vacuum oven at 2 torr and heated at $80{ }^{\circ} \mathrm{C}$ for 12 hours and then at $120^{\circ} \mathrm{C}$ for 4 hours. The catalyst concentration for the P-29 film was reduced by half to counteract the higher reactivity of the DVS monomer. The film sample for AFM measurement was cured with one side facing a silicon wafer to ensure that it is smooth.

Density determination. A piece of polymer film was first immersed in water at $25{ }^{\circ} \mathrm{C} .40 \mathrm{wt} \%$ $\mathrm{NaBr}$ solution was added until the film was exactly levitated. The density of the $\mathrm{NaBr}$ solution was then determined by a densimeter (DMA 4500 M, Anton Paar) and designated as the density of the polymer.

FT-IR spectra. Shimadzu IRTracer-100 FT-IR with ATR diamond was used to characterize P-9, P-29 and P-50 particles. Reduction of the thiol peak near $2550 \mathrm{~cm}^{-1}$ and the alkene peak near 800 $\mathrm{cm}^{-1}$ were used to monitor the reaction.

DSC measurements. DSC was performed with Discovery 2500 Differential Scanning Calorimeter. 5-10 mg of sample were prepared in aluminum hermetic pans from TA. Typical tests consist of a heat-cool-heat cycle $\left(-40^{\circ} \mathrm{C}\right.$ to $\left.120^{\circ} \mathrm{C}\right)$ at a rate of $5^{\circ} \mathrm{C} / \mathrm{min}$.

DMA measurements. Tests were conducted on a RSA-G2 dynamic mechanical analyzer (TA Instruments) equipped with Forced Convection oven $\left(20^{\circ} \mathrm{C}-\sim 500^{\circ} \mathrm{C}\right)$ attached to an Air Chiller System $\left(-120^{\circ} \mathrm{C} \sim 20^{\circ} \mathrm{C}\right)$. Samples with thickness of around $0.7 \mathrm{~mm}$ were prepared following the method described above and measured under tensile mode. The temperature was ramped at $1.5^{\circ} \mathrm{C} / \mathrm{min}$ and the oscillatory frequency used was $1 \mathrm{~Hz}$. For measuring the plasticized films, the films were soaked in their respective carrier fluid at $20{ }^{\circ} \mathrm{C}$ above $T_{\mathrm{g}}$ for 10 days and the measurement was done while immersed in the carrier fluid. For determination of the thermal 
expansion coefficients, a constant force $0.1 \mathrm{~N}$ was maintained using the isoforce mode and the length $(L)$ change with temperature $(T)$ was recorded. The linear thermal expansion coefficients were extracted by linear fitting to $\ln L$ v.s.T. $\alpha=1 / L \times d(L) / d(T)=d(\ln L) / d(T)$ in the glassy and rubbery regime.

Shear rheometry measurements. Rheological characterization was performed using an ARESG2 shear rheometer (TA Instruments) equipped with Forced Convection oven $\left(20{ }^{\circ} \mathrm{C}-\sim 500{ }^{\circ} \mathrm{C}\right)$ attached to an Air Chiller System $\left(-120^{\circ} \mathrm{C} \sim 20^{\circ} \mathrm{C}\right)$. All the rheometry data were acquired with $25 \mathrm{~mm}$ parallel plates. Change of the gap size due to temperature was calibrated beforehand. The suspension was loaded to the rheometer and extra fluid was trimmed at a gap size of $255 \mu \mathrm{m}$. The measurement gap size was maintained $240 \mu \mathrm{m}$ throughout the measurement. Temperature was equilibrated for 10 minutes before each measurement followed by a preshearing step at a stress around 2 times of the onset shear stress for 2 minutes. At each stress value, the fluid was equilibrated for a minimum of 30 seconds and averaged over 15 seconds. The first point after preshear was at least equilibrated for 105 seconds and avaraged over 15 seconds. Stress controlled measurements were done by looping a series of creep tests on the ARES-G2 rheometer. Temperature ramp experiments were carried out at a ramp rate of $1.5^{\circ} \mathrm{C} / \mathrm{min}$ for both heating and cooling.

Pull tests. MCR302 rheometer (Anton Paar) was used to measure the pull force following literature procedures ${ }^{15}$. A cylindrical metal cup with $2 \mathrm{~mm}$ depth and $10 \mathrm{~mm}$ in diameter was fixed to the bottom plate to contain the suspension. An $8 \mathrm{~mm}$ (diameter) rod that was partially embedded to a depth of $1 \mathrm{~mm}$ in the sample surface and withdrawn at a speed of $8 \mathrm{~mm} / \mathrm{s}$. Simultaneously, the normal force response on the rod was recorded. Videos were taken with a high-speed camera.

\section{Acknowledgements}

This work was supported by NIST contract 60NANB15D077, the Center for Hierarchical Materials Design (CHiMaD). Chuqiao Chen thanks Dr. Phil Griffin, Dr. Jureller, Dr. Tyler Jorgenson, Dr. Heyi Liang, Dr. Philip M. Rauscher, and Prof. Mark Ediger for discussion; the 
405 Pritzker School of Molecular Engineering for supporting her studies; her advisors and colleagues

406 for supporting each other during the covid-19 pandemic. N.D.D. thanks the Pritzker School of

407 Molecular Engineering for support through a postdoctoral fellowship. Parts of this work were

408 carried out at the Soft Matter Characterization Facility and at the Materials Research Science and

409 Engineering Center (MRSEC NSF DMR1420709) at the University of Chicago.

\section{Author contributions}

411 Chuqiao Chen, Dr. Abhinendra Singh and Dr. Neil Dolinski designed the experiments. Chuqiao

412 Chen and Michael van de Naald collected the data. Chuqiao Chen, Dr. Abhinendra Singh and Dr.

413 Grayson Jackson conceived and performed the data analysis. Dr. Heinrich M. Jaeger, Dr. Stuart

414 J. Rowan and Dr. Juan J. de Pablo supervised the whole project and provided academic advice.

415 All the authors contributed to the drafting process.

\section{Competing interests}

417 The authors have no competing/conflicting interests to declare. 


\section{References}

1. Morris, J. F. Shear thickening of concentrated suspensions: recent developments and relation to other phenomena. Annual Review of Fluid Mechanics 52, 121-144 (2020).

2. Brown, E. \& Jaeger, H. M. The role of dilation and confining stresses in shear thickening of dense suspensions. Journal of Rheology 56, 875-923 (2012).

3. Singh, A., Jackson, G. L., van der Naald, M., de Pablo, J. J. \& Jaeger, H. M. Stress-activated Constraints in Dense Suspension Rheology. 21-26 (2021).

4. Lee, Y. S., Wetzel, E. D. \& Wagner, N. J. The ballistic impact characteristics of Kevlar ${ }^{\circledR}$ woven fabrics impregnated with a colloidal shear thickening fluid. Journal of Materials Science 38, 28252833 (2003).

5. Cwalina, C. D., McCutcheon, C. M., Dombrowski, R. D. \& Wagner, N. J. Engineering enhanced cut and puncture resistance into the thermal micrometeoroid garment (TMG) using shear thickening fluid (STF) - Armor ${ }^{\mathrm{TM}}$ absorber layers. Composites Science and Technology 131, 61-66 (2016).

6. Kalman, D. P., Merrill, R. L., Wagner, N. J. \& Wetzel, E. D. Effect of particle hardness on the penetration behavior of fabrics intercalated with dry particles and concentrated particle-fluid suspensions. ACS Applied Materials and Interfaces 1, 2602-2612 (2009).

7. Gürgen, S., Kuşhan, M. C. \& Li, W. Shear thickening fluids in protective applications: A review. Progress in Polymer Science 75, 48-72 (2017).

8. Sánchez Menéndez, A. et al. Retractable intelligent speed bump. (2015).

9. Peters, I. R., Majumdar, S. \& Jaeger, H. M. Direct observation of dynamic shear jamming in dense suspensions. Nature 532, 214-217 (2016).

10. Seto, R., Mari, R., Morris, J. F. \& Denn, M. M. Discontinuous shear thickening of frictional hardsphere suspensions. Physical Review Letters 111, 1-5 (2013).

11. Royer, J. R., Blair, D. L. \& Hudson, S. D. Rheological Signature of Frictional Interactions in Shear Thickening Suspensions. Physical Review Letters 116, 1-5 (2016).

12. Singh, A., Ness, C., Seto, R., de Pablo, J. J. \& Jaeger, H. M. Shear Thickening and Jamming of Dense Suspensions: The "Roll" of Friction. Physical Review Letters 124, 248005 (2020).

13. Rathee, V., Blair, D. L. \& Urbach, J. S. Localized stress fluctuations drive shear thickening in dense suspensions. Proceedings of the National Academy of Sciences 114, 8740-8745 (2017).

14. van der Naald, M., Zhao, L., Jackson, G. L. \& Jaeger, H. M. The role of solvent molecular weight in shear thickening and shear jamming. Soft Matter 17, 3144-3152 (2021).

15. James, N. M., Han, E., de la Cruz, R. A. L., Jureller, J. \& Jaeger, H. M. Interparticle hydrogen bonding can elicit shear jamming in dense suspensions. Nature Materials 17, 965-970 (2018). 
16. Qin, J., Zhang, G. \& Shi, X. Study of a shear thickening fluid: the suspensions of monodisperse polystyrene microspheres in polyethylene glycol. Journal of Dispersion Science and Technology 38, 935-942 (2017).

17. Krieger, I. M. \& Dougherty, T. J. A Mechanism for Non-Newtonian Flow in Suspensions of Rigid Spheres. Transactions of the Society of Rheology 3, 137-152 (1959).

18. Wyart, M. \& Cates, M. E. Discontinuous shear thickening without inertia in dense non-brownian suspensions. Physical Review Letters 112, 1-5 (2014).

19. Comtet, J. et al. Pairwise frictional profile between particles determines discontinuous shear thickening transition in non-colloidal suspensions. Nature Communications 8, 1-7 (2017).

20. Brown, E. et al. Generality of shear thickening in dense suspensions. Nature Materials 9, 220-224 (2010).

21. Hsu, C. P., Mandal, J., Ramakrishna, S. N., Spencer, N. D. \& Isa, L. Exploring the roles of roughness, friction and adhesion in discontinuous shear thickening by means of thermo-responsive particles. Nature Communications 12, 1-10 (2021).

22. Hsu, C. P., Ramakrishna, S. N., Zanini, M., Spencer, N. D. \& Isa, L. Roughness-dependent tribology effects on discontinuous shear thickening. Proceedings of the National Academy of Sciences of the United States of America 115, 5117-5122 (2018).

23. Mueller, S., Llewellin, E. W. \& Mader, H. M. The rheology of suspensions of solid particles. Proceedings of the Royal Society A: Mathematical, Physical and Engineering Sciences 466, 12011228 (2010).

24. Singh, A., Mari, R., Denn, M. M. \& Morris, J. F. A constitutive model for simple shear of dense frictional suspensions. Journal of Rheology 62, 457-468 (2018).

25. Singh, A., Pednekar, S., Chun, J., Denn, M. M. \& Morris, J. F. From yielding to shear jamming in a cohesive frictional suspension. Physical Review Letters 122, 98004 (2019).

26. Fakhraai, Z. \& Forrest, J. A. Measuring the surface dynamics of glassy polymers. Science $\mathbf{3 1 9}, 600-$ 604 (2008).

27. Ediger, M. D. \& Forrest, J. A. Dynamics near free surfaces and the glass transition in thin polymer films: a view to the future. Macromolecules 47, 471-478 (2014).

28. Ediger, M. D., Angell, C. A. \& Nagel, S. R. Supercooled liquids and glasses. J. Phys. Chem. V, 13200-13212 (1996).

29. Chen, N., Maeda, N., Tirrell, M. \& Israelachvili, J. Adhesion and friction of polymer surfaces: The effect of chain ends. Macromolecules 38, 3491-3503 (2005).

30. Maeda, N., Chen, N., Tirrell, M. \& Israelachvili, J. N. Adhesion and friction mechanisms of polymer-on-polymer surfaces. Science 297, 379-382 (2002). 
31. Chen, Y. L., Helm, C. A. \& Israelachvili, J. N. Molecular mechanisms associated with adhesion and contact angle hysteresis of monolayer surfaces. Journal of Physical Chemistry 95, 10736-10747 (1991).

32. Zeng, H., Maeda, N., Chen, N., Tirreh, M. \& Israelachvili, J. Adhesion and friction of polystyrene surfaces around Tg. Macromolecules 39, 2350-2363 (2006).

33. Sills, S., Gray, T. \& Overney, R. M. Molecular dissipation phenomena of nanoscopic friction in the heterogeneous relaxation regime of a glass former. The Journal of Chemical Physics 123, 134902 (2005).

34. Knorr, D. B., Gray, T. O. \& Overney, R. M. Cooperative and submolecular dissipation mechanisms of sliding friction in complex organic systems. The Journal of Chemical Physics 129, 074504 (2008).

35. Bueche, A. M. \& Flom, D. G. Surface friction and dynamic mechanical properties of polymers.

36. Krieger, I. M. Rheology of monodisperse latices. Advances in Colloid and Interface Science 3, 111136 (1972).

37. Ugǔr, Ş. \& Pekcan, Ö. The effect of annealing temperature on latex film dissolution. Journal of Colloid and Interface Science 277, 359-365 (2004).

38. Goh, M. C., Juhué, D., Leung, O. M., Wang, Y. \& Winnik, M. A. Annealing effects on the surface structure of latex films studied by atomic force microscopy. Langmuir 9, 1319-1322 (1993).

39. Shim, S. E., Yang, S., Jung, H. \& Choe, S. Thermally robust highly crosslinked poly(methyl methacrylate-co-divinyl benzene) microspheres by precipitation polymerization. Macromolecular Research 12, 233-239 (2004).

40. Loshaek, S. Crosslinked polymers. II. Glass temperatures of copolymers of methyl methacrylate and glycol dimethacrylates. Journal of Polymer Science 15, 391-404 (1955).

41. Cha, Y.-J. \& Choe, S. Characterization of crosslinked polystyrene beads and their composite in SBR matrix. Journal of Applied Polymer Science 58, 147-157 (1995).

42. Wang, C., Podgórski, M. \& Bowman, C. N. Monodisperse functional microspheres from stepgrowth "click" polymerizations: preparation, functionalization and implementation. Materials Horizons 1, 535-539 (2014).

43. Wang, C. et al. Monodispersity/narrow polydispersity cross-linked microparticles prepared by step-growth thiol-Michael addition dispersion polymerizations. Macromolecules 48, 8461-8470 (2015).

44. Nair, D. P. et al. The Thiol-Michael addition click reaction: A powerful and widely used tool in materials chemistry. Chemistry of Materials vol. 26 724-744 (2014).

45. Schroeter, J. \& Hobelsberger, M. On the mechanical properties of native Starch Granules. Starch Stärke 44, 247-252 (1992). 
46. Cavaille, J. Y., Jourdan, C., Perez, J., Monnerie, L. \& Johari, G. P. Time-temperature superposition and dynamic mechanical behavior of atactic polystyrene. Journal of Polymer Science Part B: Polymer Physics 25, 1235-1251 (1987).

47. Davis, W. M. \& Macosko, C. W. Nonlinear dynamic mechanical moduli for polycarbonate and PMMA. Journal of Rheology 22, 53-71 (1978).

48. Carlotti, G., Doucet, L. \& Dupeux, M. Elastic properties of silicon dioxide films deposited by chemical vapour deposition from tetraethylorthosilicate. Thin Solid Films 296, 102-105 (1997).

49. Tian, T., Peng, G., Li, W., Ding, J. \& Nakano, M. Experimental and modelling study of the effect of temperature on shear thickening fluids. Korea-Australia Rheology Journal 27, 17-24 (2015).

50. Maranzano, B. J. \& Wagner, N. J. The effects of particle size on reversible shear thickening of concentrated colloidal dispersions. The Journal of Chemical Physics 114, 10514-10527 (2001).

51. Roth, C. B. Polymer glasses Ch.1-4. (CRC Press, Boca Raton, 2016).

52. Jamali, S., Boromand, A., Wagner, N. \& Maia, J. Microstructure and rheology of soft to rigid shear-thickening colloidal suspensions. Journal of Rheology 59, 1377-1395 (2015).

53. Osswald, T. \& Rudolph, N. Polymer Rheology: Fundamentals and Applications Ch.5. Hanser Publications vol. 37 (Hanser Publications, Munich, 2015).

54. Zahoranová, A., Mrlík, M., Tomanová, K., Kronek, J. \& Luxenhofer, R. ABA and BAB triblock copolymers based on 2-methyl-2-oxazoline and 2- $\mathrm{n}$-propyl-2-oxazoline: synthesis and thermoresponsive behavior in water. Macromolecular Chemistry and Physics 218, 1700031 (2017).

55. Tam, K. C., Wu, X. Y. \& Pelton, R. H. Viscometry-a useful tool for studying conformational changes of poly(N-isopropylacrylamide) in solutions. Polymer 33, 436-438 (1992).

56. Rubinstein, M. \& Colby, R. H. Polymer Physics Ch. 8-9. (Oxford University Press, Oxford, 2003).

57. Smith, M. I., Besseling, R., Cates, M. E. \& Bertola, V. Dilatancy in the flow and fracture of stretched colloidal suspensions. Nature Communications 1, 114 (2010). 


\section{Supplementary Files}

This is a list of supplementary files associated with this preprint. Click to download.

- P50suspensionpulltest.mp4

- SITgjammingv211123.pdf

- P9suspensionpulltest.mp4 